\title{
Aspirin for Prevention of Preeclampsia in Lupus Pregnancy
}

\author{
Amelie M. Schramm ${ }^{1}$ and Megan E. B. Clowse ${ }^{2}$ \\ ${ }^{1}$ Department for Internal Medicine 3 and Institute for Clinical Immunology, Friedrich-Alexander-University \\ Erlangen-Nuremberg, Erlangen, Germany \\ ${ }^{2}$ Department of Medicine, Duke University Medical Center, P.O. Box 3535, Trent Drive, Durham, NC 27710, USA
}

Correspondence should be addressed to Megan E. B. Clowse; megan.clowse@duke.edu

Received 4 September 2013; Revised 6 December 2013; Accepted 9 December 2013; Published 20 March 2014

Academic Editor: Ricard Cervera

Copyright (c) 2014 A. M. Schramm and M. E. B. Clowse. This is an open access article distributed under the Creative Commons Attribution License, which permits unrestricted use, distribution, and reproduction in any medium, provided the original work is properly cited.

\begin{abstract}
Preeclampsia, the onset of hypertension and proteinuria during pregnancy, is a common medical disorder with high maternal and fetal mortality and morbidity. The underlying pathology remains poorly understood and includes inflammation, endothelial dysfunction, and an unbalanced thromboxane $\mathrm{A}_{2}$ /prostacyclin ratio. For women with systemic lupus erythematosus (SLE), particularly those with preexisting renal disease or with active lupus, the risk of developing preeclampsia is up to $14 \%$ higher than it is among healthy individuals. The mechanism is still unknown and the data for preventing preeclampsia in lupus pregnancies are rare. Modulating the impaired thromboxane $\mathrm{A}_{2}$ /prostacyclin ratio by administration of low-dose aspirin appears to be the current best option for the prevention of preeclampsia. After providing an overview of the pathogenesis of preeclampsia, preeclampsia in lupus pregnancies, and previous trials for prevention of preeclampsia with aspirin treatment, we recommend low-dose aspirin administration for all lupus patients starting prior to 16 weeks of gestation. Patients with SLE and antiphospholipid syndrome should receive treatment with heparin and low-dose aspirin during pregnancy.
\end{abstract}

\section{Introduction}

Preeclampsia is defined by an increase in blood pressure $(>140 / 90)$ and proteinuria $(>300 \mathrm{mg} / 24 \mathrm{hr})$ in the latter half of pregnancy. While modern obstetrical management has made it less dangerous than before, it is still associated with an increased rate of maternal and fetal mortality and is an important cause for preterm birth [1]. It is difficult to identify pregnancies at particularly high risk for preeclampsia before it presents clinically; however, there are several known risk factors. These include first birth, a first birth with a new father, prior hypertension or renal disease, diabetes, and prior preeclampsia. Both systemic lupus erythematosus (SLE) and antiphospholipid syndrome (APS) put pregnancies at high risk for this complication. Uterine Doppler studies can identify pregnancies with particularly high uterine artery pressures, which may be an indicator of early placental changes that lead to preeclampsia [1]. The goal of this paper is to provide insight into the mechanisms through which SLE and APS contribute to preeclampsia and the potential role that low-dose aspirin may play in mitigating this risk.

\section{Pathogenesis}

2.1. Pathophysiology of Preeclampsia. As a common medical disorder during pregnancy, preeclampsia causes hypertension (systolic blood pressure $>140 \mathrm{mmHg}$ or diastolic blood pressure $>90 \mathrm{mmHg}$ ), proteinuria $(>300 \mathrm{mg} / 24 \mathrm{~h}$ ), and in rare cases additional symptoms like hyperreflexia, seizures (eclampsia), acute renal failure, pulmonary complications, the triad of hemolysis, elevated liver enzymes, and low platelets (HELLP syndrome).

The disease typically starts after 20 weeks of gestation and is an important factor for maternal and fetal mortality and morbidity. In the United States, around $5-8 \%$ of pregnant women are affected [2]. The only definitive treatment is delivery of the baby. While preeclampsia's underlying pathology remains poorly understood, the onset of this condition 


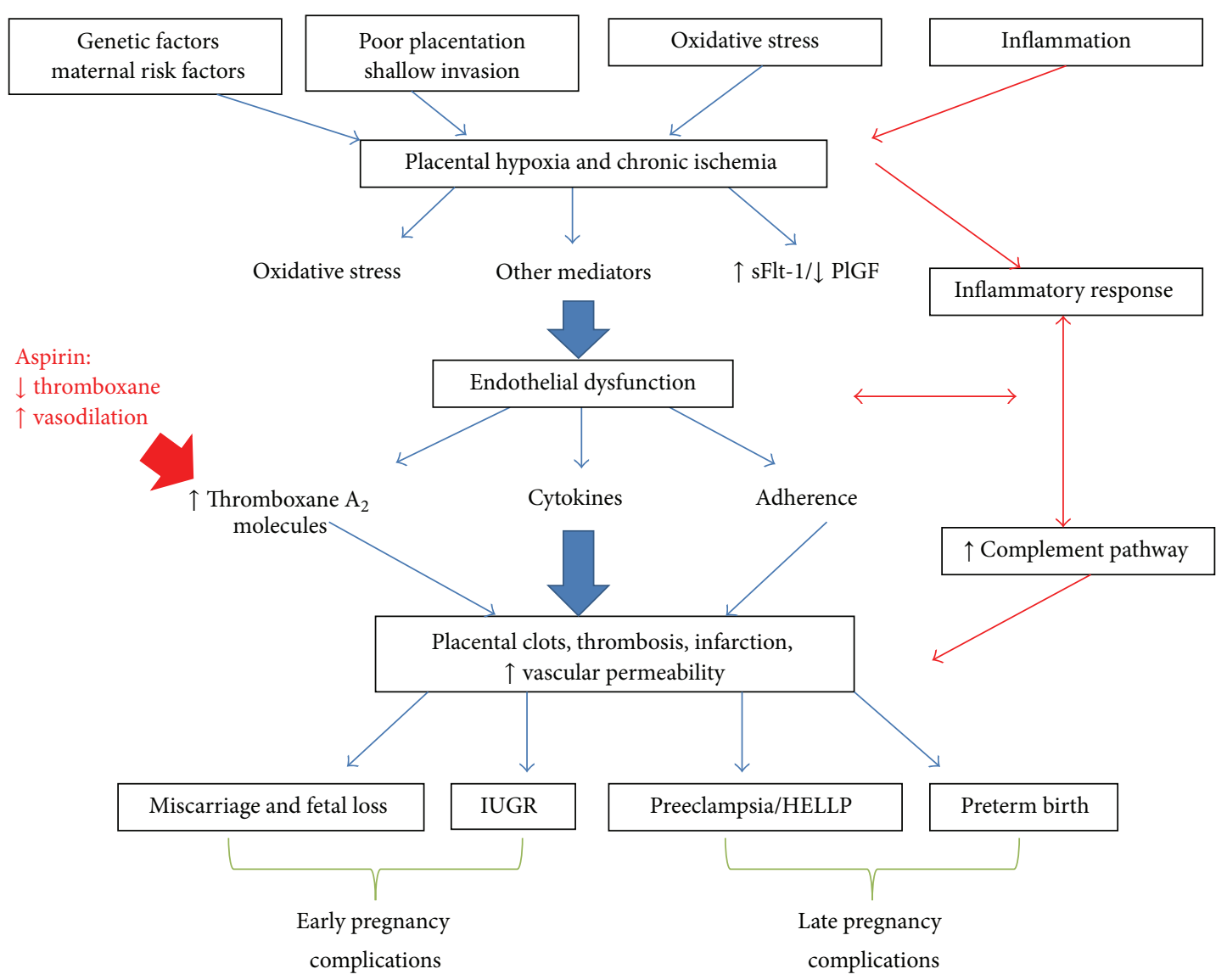

FIGURE 1: Mechanisms of preeclampsia.

involves shallow trophoblast invasion with poor placentation, inflammation, dysregulation of angiogenic factors, and ischemia, all of which lead to the central mechanism of endothelial dysfunction. Endothelial dysfunction causes, among other things, activation of platelets, a rise in thromboxane levels, and an ensuing clotting cascade (Figure 1).

2.1.1. Poor Placental Vascular Remodeling. Implantation and placental development happen in the first trimester of pregnancy. Fetal cytotrophoblasts invade the maternal spiral arteries and convert these high-resistance, muscular arteries to high-capacitance, elastic vessels. Insufficient spiral artery transformation is strongly associated with the pathology of severe preeclampsia. In preeclamptic placentas, shallow trophoblast invasion prevents the necessary vascular remodeling, which leads to decreased perfusion, hypoxia, and chronic placental ischemia [3].

2.1.2. Imbalance of Angiogenic Factors. Chronic ischemia caused by poor vascularization is associated with the placental production of angiogenic factors like vascular endothelial growth factor (VEGF), placental growth factor (PlGF), and soluble fms-like tyrosine kinase-1 (sFlt-1). VEGF encourages the growth of blood vessels, supports proper function of endothelial cells, and stimulates NO production in vascular walls $[4,5]$. SFlt-1 is a naturally occurring VEGF antagonist which binds free VEGF and occupies the VEGF receptor [6]. Experiments and animal models suggest that VEGF and sFlt1 may play a role in the pathogenesis of preeclampsia [7]. During healthy pregnancies, levels of placental growth factor (PlGF) increase in the first and second trimesters and fall in the third trimester. The level of antiangiogenic factor sFlt1 usually remains steady during the first part of pregnancy and rises during the last trimester. In blood samples of patients with preeclampsia, however, lower levels of PlGF throughout gestation and increased levels of sFlt-1 levels at 26 and 29 weeks of gestation are detectable [8-10]. An elevated sFlt-1/PlGF ratio during the second trimester, but not the first trimester, can help detect preeclampsia even before the presentation of clinical symptoms [11-13]. Increased levels of sFlt-1 also can be found in lupus patients with preeclampsia [14].

2.1.3. Inflammation. Systemic maternal inflammation is also involved in the pathogenesis of preeclampsia. Levels of circulating proinflammatory mediators like IL-6, IL-8, TNF$\alpha$, and monocyte chemoattractant protein 1 (MCP-1) are markedly higher in preeclamptic pregnancies than in healthy pregnancies [19]. Noninfectious leukocyte infiltration of the villi (fetal) and the decidua (maternal interface) was found in preeclamptic placentas, along with an uncontrolled, increased activation of the complement system, with elevated 
levels of complement-activation factor Bb. Activation of the complement system is commonly seen as an important mechanism linking inflammation and coagulation [20]. Inflammation may therefore be a trigger for the development of preeclampsia in patients with SLE [21].

2.1.4. Thromboxane. Poor placental perfusion leads to activation of platelets and the clotting cascade, resulting in an imbalance among vasoactive prostaglandins. The ratio between the prostaglandins thromboxane and prostacyclin modulates vascular blood flow, with thromboxane $\mathrm{A}_{2}$ acting as a vasoconstrictor and promoting platelet aggregation, while prostacyclin acts as a vasodilator and inhibits aggregation. Increased thromboxane and reduced prostacyclin levels are associated with infarction and thrombotic vasculopathy, which are well-known features in preeclamptic placentas [22].

The constitutive enzyme cyclooxygenase 1 produces thromboxane $\mathrm{A}_{2}$ in platelets and primarily prostacyclin in endothelial cells. Aspirin, a common irreversible inhibitor of cyclooxygenases, acts particularly in platelets. The impact of aspirin use in preeclampsia prevention is shown by data which demonstrate an aspirin-induced decrease of thromboxane concentration and mediation of the unbalanced thromboxane $\mathrm{A}_{2}$ /prostacyclin ratio [23] (Figure 2). Aspirin thus improves placental blood flow and minimizes risk of placental thrombosis, which serves as the rationale for administering prophylactic low-dose aspirin for prevention of preeclampsia.

2.2. Pregnancy in Patients with Systemic Lupus Erythematosus. Systemic lupus erythematosus (SLE) is a pervasive autoimmune disease which can affect nearly every organ and tissue in the body with an extremely broad variability in severity. Women with SLE are often diagnosed in their childbearing years [24]. As SLE usually has no influence on female fertility, pregnancies are common among these women [25].

Maternal and fetal risk for serious medical and pregnancy complications is significantly higher for women with SLE than for healthy women. National analysis showed a 20fold higher risk for maternal mortality among lupus patients, who are also at increased risk for preterm labor (OR 2.4), Cesarean section (OR 1.7), and fetal growth restriction (OR 2.6) [26]. Compared to healthy individuals, women with SLE, especially those with preexisting renal disease or with active SLE before and during pregnancy, have a higher risk for developing preeclampsia. Up to $30 \%$ of all lupus pregnancies are complicated by preeclampsia $[27,28]$.

While active lupus is the main predictor for pregnancy complications, other identified risk factors are strongly associated with preeclampsia, including preexisting hypertension, antiphospholipid syndrome (APS), obesity, positive anti-double-stranded DNA antibodies (dsDNA) or antiribonucleoprotein (RNP) antibodies, low complement [2], and thrombocytopenia at onset of pregnancy [29]. Thrombocytopenia that occurs in lupus pregnancy before 15 weeks of gestation is usually due to SLE activity (platelet-specific antibodies) or APS. After 25 weeks, low platelet counts are more commonly caused by preeclampsia/HELLP syndrome [27].
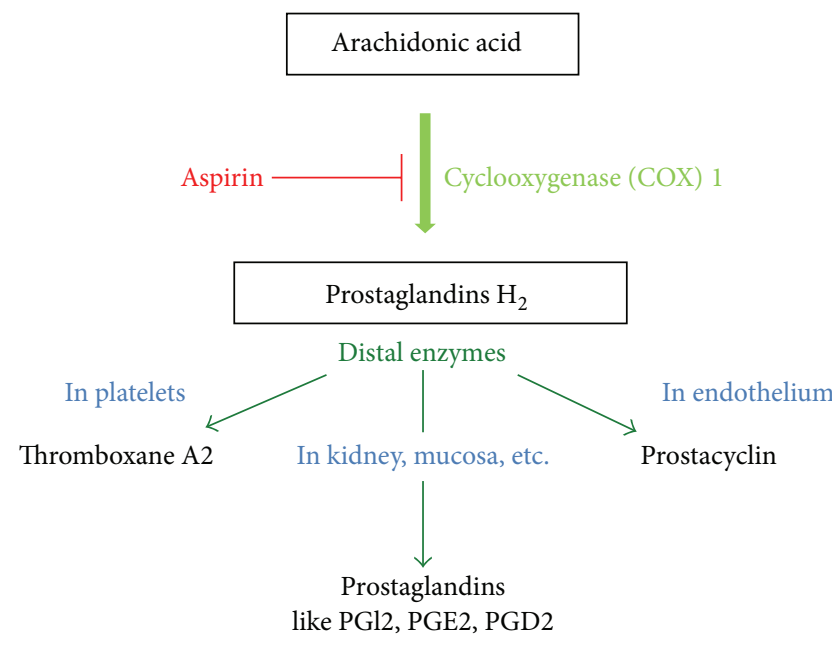

FIGURE 2: Interaction of aspirin with COX 1.

Differentiating between lupus activity and pregnancyrelated complications presents a major challenge in the management of SLE pregnancies. In both SLE and preeclampsia, for example, women develop worsening proteinuria with hypertension and edema. Yet it remains essential to accurately determine whether symptoms are due to lupus or to preeclampsia, given the different treatments required for preeclampsia (delivery) and SLE (immunosuppression) [2]. Decreased levels of complement and active urine sediment could suggest lupus nephritis, whereas elevated serum uric acid and low urine calcium are more typical for preeclampsia. Additionally, the presence of concomitant lupus symptomslike arthritis, serositis, skin lesions, or rising levels of dsDNAantibodies-could point to SLE activity.

While women who experience increased SLE activity during pregnancy are the most at risk, even those with quiescent lupus are more likely to develop preeclampsia than healthy women. This suggests that SLE and preeclampsia may share a common underlying pathology.

2.2.1. Endothelial Dysfunction. Chronic autoimmune diseases with systemic inflammation lead to an increased risk for cardiovascular disease (CVD), with atherosclerosis and vascular alterations through changes in vascular adhesion molecules, increased transendothelial permeability, impaired antithrombotic properties, and reduced production of vasodilators and thrombomodulin expression. Thrombomodulin limits aggregation of platelets and activation of the complement pathway. The presence of proinflammatory cytokines, however, lowers thrombomodulin and increases levels of the procoagulant tissue factor [30].

Impaired endothelial repair is also linked to endothelial dysfunction in SLE. While patients with SLE have similar numbers of endothelial progenitor cells compared to healthy people, these cells exhibit impaired migratory and adhesive properties [31]. We suggest that the endothelial dysfunction inherent in SLE may contribute to the risk of preeclampsia in this population. 
TABLE 1: Treatment of anti-phospholipid antibodies in pregnancy.

\begin{tabular}{ll}
\hline Clinical presentation & Suggested treatment \\
\hline $\begin{array}{l}\text { Patients with aPL and no history of thrombosis and no } \\
\text { series of fetal loss or early delivery due to preeclampsia } \\
\text { or placental insufficiency }\end{array}$ & Addition of low-dose aspirin throughout pregnancy \\
\hline $\begin{array}{l}\text { Patients with APS and no history of thrombosis } \\
\text { but with previous historyof stillbirth, recurrent fetal } \\
\text { loss, or other APS-associated pregnancy complications }\end{array}$ & $\begin{array}{l}\text { Heparin or LMWH (usual prophylactic dose) during } \\
\text { pregnancy and 6 weeks postpartum } \\
\text { Low-dose aspirin throughout pregnancy }\end{array}$ \\
\hline $\begin{array}{l}\text { Patients with APS and prior history of thrombosis or } \\
\text { embolism }\end{array}$ & $\begin{array}{l}\text { Heparin or LMWH (usual therapeutic dose) during } \\
\text { pregnancy and 6 weeks postpartum followed by } \\
\text { optional conversion on warfarin } \\
\text { Low-dose aspirin throughout pregnancy }\end{array}$ \\
\hline
\end{tabular}

2.2.2. Inflammation. During pregnancy, T-cells play an important role in modulating the maternal immune system as it adapts to a semiallogeneic fetus [32]. Fewer regulatory Tcells (Treg) and increased T helper-17 cell (Th17) activity have been found in women with preeclampsia. Similar changes are common in active SLE. One study of SLE pregnancies, for example, documented particularly low levels of Treg cells in the context of preeclampsia [33-36]. We suggest that the immune dysregulation seen in SLE may contribute to the risk of preeclampsia. Reduced systemic inflammation and normalized T-cell activity during lupus quiescence may lead to a reduction of pregnancy complications in the absence of lupus activity.

2.3. Antiphospholipid Syndrome. Antiphospholipid syndrome (APS) is a prothrombotic disorder which can cause thrombosis, embolism, or stroke and is highly associated with pregnancy complications like miscarriage and preeclampsia. The antibodies of APS are circulating anti-phospholipid antibodies (aPL), which are characterized as anti-cardiolipin antibodies (aCL), lupus-anticoagulant (LAC), and $\beta 2$-glycoprotein antibodies (anti- $\beta 2-\mathrm{GPI}$ ). Patients are diagnosed by the combination of detectable antibodies and clinical findings (including thrombosis, embolism, and stroke) or obstetrical failures (including three sequential early pregnancy losses, a second- or third-trimester loss, or severe early preeclampsia).

2.3.1. APS and Pregnancy. The risk for preeclampsia is more than ninefold higher in APS patients than in healthy women [37]. The pregnancy complications of APS appear to result from the interaction of prothrombotic factors, inflammation, and trophoblast pathologies. Phospholipid-binding proteins-annexin V, protein $\mathrm{C}$, prothrombin, or anti- $\beta 2-\mathrm{GPI}-$ are antigen targets for aPL. Whereas most human cells translocate $\beta 2$-glycoprotein on their surface only during apoptosis or pathological conditions, trophoblasts continually present $\beta 2$-GPI on their cell membranes. This could explain $\beta 2$-GPI placental tropism and placenta-related pregnancy complications in women with APS [38].

Clots, thrombosis, and placental infarction due to the presence of antibodies and the following platelet activation are common in APS placentas. While aPL does not react with resting endothelial cells, Chen et al. found that a triggering event such as phagocytosis of necrotic trophoblastic debris allows aPL to influence endothelial cells for a prolonged period. After a trigger event, aPL can maintain activation of endothelial cells even without the further presence of necrotic trophoblastic debris. Chronic activation of the endothelium leads to an imbalance between thromboxane $\mathrm{A}_{2}$ and prostacyclin [39]. Correction of this imbalance by low-dose aspirin may explain the benefit of aspirin administration in APS pregnancy.

Pregnant women with anti-phospholipid antibodies are at risk for catastrophic APS (CAPS), which presents with rapid evolution of thrombosis, often microthrombi, in 3 or more organs. CAPS is deadly: up to half of women who develop this in pregnancy die, as do half of the infants. The role that aspirin might play in the prevention or treatment of CAPS is unclear, but aggressive anticoagulation, plasmapheresis, and immunosuppression are all suggested therapies. CAPS may present concurrently with preeclampsia and/or HELLP (hemolysis, elevated liver enzymes, and low platelets) syndrome [40].

Treatment guidelines suggest combining therapies of low-dose aspirin and heparin for all APS patients during pregnancy (Table 1). Depending on the patient's history of thrombosis, miscarriage, or other pregnancy complications, the medication may be extended to anticoagulation with full therapeutic dose $[41,42]$.

Studies have documented a decrease in pregnancy loss among patients with APS who receive heparin treatment, but heparin does not appear to be as effective in preventing late pregnancy complications such as preeclampsia [42]. In addition to its anticoagulant properties, heparin can interrupt the interaction between aPL and $\beta 2-\mathrm{GPI}$, inhibit the complement pathway, and provide supplemental antiinflammatory effects, but it does not influence trophoblast migration or placentation. This may explain heparin's lack of significant benefit for prevention of late pregnancy complications like preeclampsia, despite its effective prevention of early pregnancy problems in APS patients [38]. Aspirin, on the other hand, may play a role in preventing late pregnancy complications. 


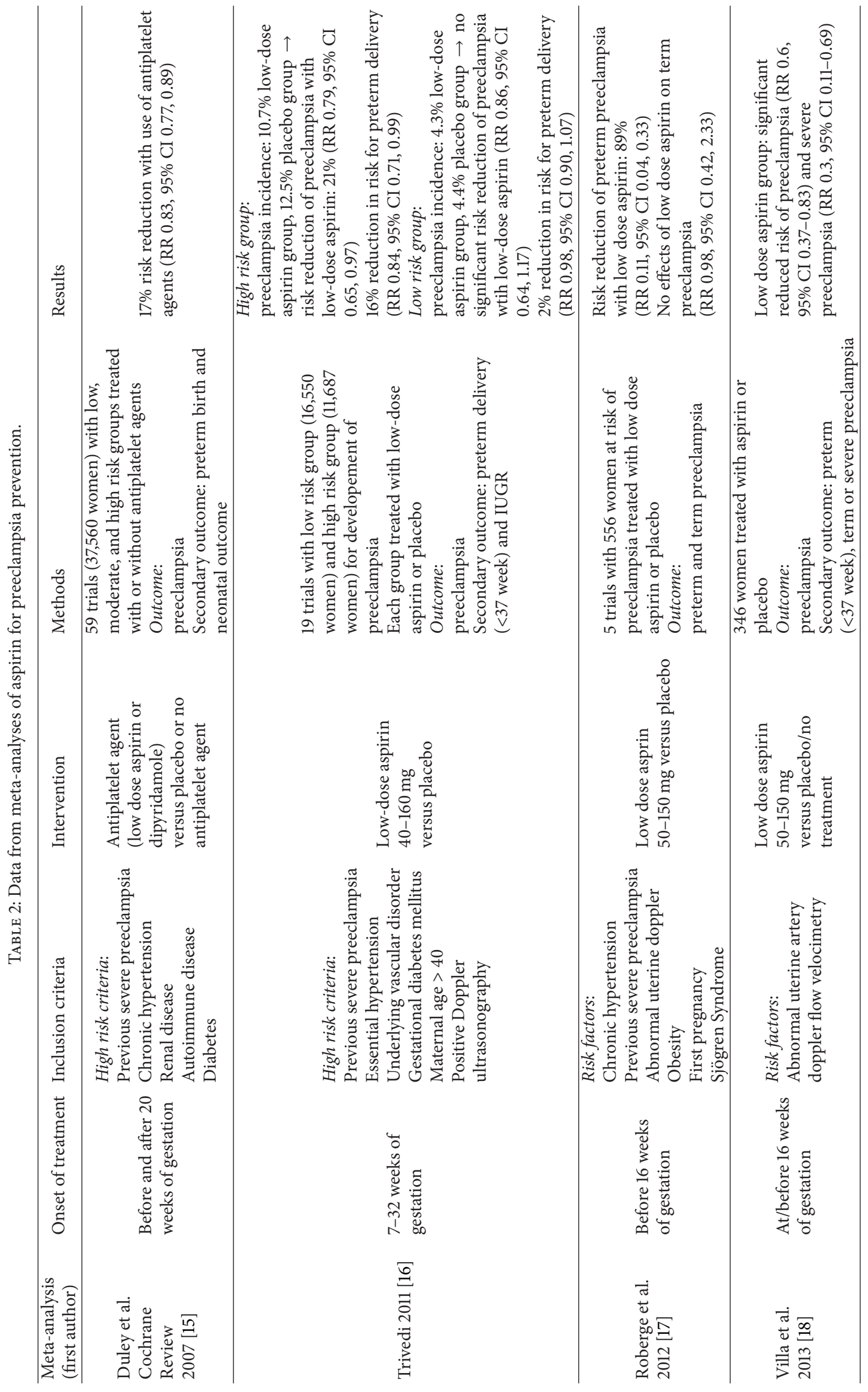




\section{Clinical Trials of Low-Dose Aspirin Administration for Prevention of Preeclampsia}

Until recently, preeclampsia has been resistant to preventive treatment. Low-dose aspirin, however, has shown beneficial effects in a wide range of clinical trials for prevention of placenta-associated pregnancy complications. While the definition of high risk for preeclampsia has not been entirely consistent between studies, patients identified as high risk typically have a history of preeclampsia or fetal growth restriction, abnormal uterine artery Doppler, essential hypertension, obesity, and/or diabetes mellitus. A few studies also included women with underlying vascular disorders or autoimmune diseases, but the number of patients studied with rheumatologic disease is very small.

Two meta-analyses demonstrated beneficial effects of aspirin for prevention of preeclampsia: among women at high risk for this complication, antiplatelet treatment reduced the risk by $17-21 \%$ (Table 2 ) $[15,16]$. In Trivedi's analysis, the incidence of preeclampsia in the high-risk group was $10.7 \%$ with low-dose aspirin administration and $12.5 \%$ with placebo administration ( $R R 0.79, P=0.02$ ). The low-risk group, however, showed no significant differences in preeclampsia incidence with aspirin (4.3\%) and with placebo (4.4\%, RR 0.86, $P=0.35$ ). These meta-analyses included fairly heterogeneous studies, with low-dose aspirin initiation ranging from 7 to 32 weeks of gestation.

More robust findings emerged from two meta-analyses that were restricted to studies in which low-dose aspirin was started prior to 16 weeks of gestation. One analysis found that while low-dose aspirin introduced $<16$ weeks of gestation decreased the risk for severe preeclampsia, perinatal death, and fetal growth restriction, low-dose aspirin initiation after 16 weeks of gestation did not provide this protective effect [17]. Villa et al. demonstrated significantly reduced risk for preeclampsia (RR 0.6, CI 0.27-0.83) and severe preeclampsia (RR 0.3, CI 0.11-0.69) with low-dose aspirin administration in women with abnormal uterine artery flow [18]. Roberge et al. found that low-dose aspirin administration resulted in an $89 \%$ risk reduction for preterm preeclampsia but did not decrease risk for term preeclampsia. Based on this data, it appears that early initiation of low-dose aspirin is important and that it may be most effective in preventing preterm and severe preeclampsia [17].

The range of aspirin dosages with positive effects appears to be quite flexible. Across studies, the administered low-dose aspirin dose was between 40 and $160 \mathrm{mg} /$ day, yet there was no difference in potency [16]. Previous trials also investigated the maternal and neonatal outcomes of pregnancies exposed to low-dose aspirin, and treatment appears to be safe for both mother and newborn $[15,43]$. Compared to women in the control groups, for example, pregnant women treated with low-dose aspirin experienced no significant difference in risk of maternal or neonatal bleeding [16]. Case-controlled data also showed no increased risk of congenital abnormalities [44]. And, unlike high-dose NSAIDs, low-dose aspirin does not appear to increase the risk for ductus arteriosus closure in utero [45]. Analysis for preconceptional low-dose aspirin administration for prevention of preeclampsia after in vitro fertilization (IVF) found no significant reduction of hypertensive pregnancy complications compared to placebo group [22].

\section{Conclusion: Impact of Anticoagulation for Prevention of Preeclampsia in Lupus Patients}

The task of lowering the risk for preeclampsia in women with SLE is challenging. Maintaining SLE quiescence may reduce this risk by minimizing the impact of chronic inflammation, but endothelial dysfunction due to systemic lupus is not currently amenable to treatment. Aspirin, however, can interfere with the subsequent pathological process of a vasoconstrictive, procoagulant, and platelet-activating state and may prevent preeclampsia by modulating the thromboxane $\mathrm{A}_{2}$ /prostacyclin ratio to optimize placental blood flow and prevent placental thrombosis.

While aspirin will not eliminate all cases of preeclampsia, it is currently the best and safest available drug for influencing the pathogenesis and clinical presentation of preeclampsia. Although there is no trial evidence for the use of low-dose aspirin to prevent preeclampsia in SLE, aspirin could lower the risk for lupus patients to an extent comparable to the risk reduction demonstrated in trials assessing low-dose aspirin treatment for other high-risk groups. Therefore, we would expect aspirin treatment to offer a risk reduction of up to $20 \%$ for preeclampsia development in lupus patients, suggesting the possibility of lowering preeclampsia incidence from $15 \%$ in all lupus pregnancies to around $12 \%$ in lupus patients with low-dose aspirin treatment.

4.1. Preeclampsia Prophylaxis in Lupus Pregnancies. Based on the pathogenic role of thromboxane in placental perfusion and the higher incidence of preeclampsia in SLE pregnancy, we recommend low-dose aspirin administration for all pregnant women with SLE, with therapy being initiated prior to 16 weeks of gestation and continuing throughout pregnancy. Women with SLE and APS should continue aspirin treatment as a preeclampsia prophylaxis and add heparin or LMWH. For pregnant women without a history of thrombosis, lower prophylactic dosing of heparin or LMWH is appropriate, but for pregnant women with a history of thrombosis, heparin administration should be increased to a full antithrombotic dose.

A future clinical trial of the use of aspirin as preeclampsia prevention in SLE should be performed to generate more exact recommendations.

\section{Conflict of Interests}

Megan E. B. Clowse serves as a consultant for UCB but on issues separate from those discussed in this paper. Schramm has no conflict of interests. 


\section{References}

[1] A. Shennan, "Preeclampsia and non-proteinuric pregnancyinduced hypertension," in Obstetrica and Gynaenocology: An Evidence-Based Text for MRCOG, D. M. Luesley and P. N. Baker, Eds., pp. 179-186, Arnold, London, UK, 2004.

[2] M. E. B. Clowse, "Lupus activity in pregnancy," Rheumatic Disease Clinics of North America, vol. 33, no. 2, pp. 237-252, 2007.

[3] L. Ji, J. Brkić, M. Liu, G. Fu, C. Peng, and Y. L. Wang, "Placental trophoblast cell differentiation: physiological regulation and pathological relevance to preeclampsia," Molecular Aspects of Medicine, vol. 34, no. 5, pp. 981-1023, 2012.

[4] I. E. Stillman and S. A. Karumanchi, "The glomerular injury of preeclampsia," Journal of the American Society of Nephrology, vol. 18, no. 8, pp. 2281-2284, 2007.

[5] G. Valdés and J. Corthorn, "Review: the angiogenic and vasodilatory utero-placental network," Placenta, vol. 32, supplement 2, pp. S170-S175, 2011.

[6] G. S. Di Marco, S. Reuter, U. Hillebrand et al., "The soluble VEGF receptor sFlt1 contributes to endothelial dysfunction in CKD," Journal of the American Society of Nephrology, vol. 20, no. 10, pp. 2235-2245, 2009.

[7] A. Bergmann, S. Ahmad, M. Cudmore et al., "Reduction of circulating soluble Flt-1 alleviates preeclampsia-like symptoms in a mouse model," Journal of Cellular and Molecular Medicine, vol. 14, no. 6 B, pp. 1857-1867, 2010.

[8] F. T. H. Wu, M. O. Stefanini, F. M. Gabhann, C. D. Kontos, B. H. Annex, and A. S. Popel, "A systems biology perspective on sVEGFR1: its biological function, pathogenic role and therapeutic use," Journal of Cellular and Molecular Medicine, vol. 14, no. 3, pp. 528-552, 2010.

[9] C. Hirashima, A. Ohkuchi, S. Matsubara et al., "Alteration of serum soluble endoglin levels after the onset of preeclampsia is more pronounced in women with early-onset," Hypertension Research, vol. 31, no. 8, pp. 1541-1548, 2008.

[10] R. Romero, J. K. Nien, J. Espinoza et al., "A longitudinal study of angiogenic (placental growth factor) and anti-angiogenic (soluble endoglin and soluble vascular endothelial growth factor receptor-1) factors in normal pregnancy and patients destined to develop preeclampsia and deliver a small for gestational age neonate," Journal of Maternal-Fetal and Neonatal Medicine, vol. 21, no. 1, pp. 9-23, 2008.

[11] J. H. Lim, S. Y. Kim, S. Y. Park, J. H. Yang, M. Y. Kim, and H. M. Ryu, "Effective prediction of preeclampsia by a combined ratio of angiogenesis-related factors," Obstetrics and Gynecology, vol. 111, no. 6, pp. 1403-1409, 2008.

[12] M. Jacobs, N. Nassar, C. L. Roberts, R. Hadfield, J. M. Morris, and A. W. Ashton, "Levels of soluble fms-like tyrosine kinase one in first trimester and outcomes of pregnancy: a systematic review," Reproductive Biology and Endocrinology, vol. 9, article 77, 2011.

[13] A. O. Odibo, C. C. Rada, A. G. Cahill et al., "First-trimester serum soluble fms-like tyrosine kinase-1, free vascular endothelial growth factor, placental growth factor and uterine artery Doppler in preeclampsia," Journal of Perinatology, vol. 33, no. 9, pp. 670-674, 2013.

[14] U. Qazi, C. Lam, S. A. Karumanchi, and M. Petri, "Soluble Fmslike tyrosine kinase associated with preeclampsia in pregnancy in systemic lupus erythematosus," Journal of Rheumatology, vol. 35, no. 4, pp. 631-634, 2008.

[15] L. Duley, D. J. Henderson-Smart, S. Meher, and J. F. King, "Antiplatelet agents for preventing pre-eclampsia and its complications," Cochrane Database of Systematic Reviews, no. 2, Article ID CD004659, 2007.
[16] N. A. Trivedi, "A meta-analysis of low-dose aspirin for prevention of preeclampsia," Journal of Postgraduate Medicine, vol. 57, no. 2, pp. 91-95, 2011.

[17] S. Roberge, P. Villa, K. Nicolaides et al., "Early administration of low-dose aspirin for the prevention of preterm and term preeclampsia: a systematic review and meta-analysis," Fetal Diagnosis and Therapy, vol. 31, no. 3, pp. 141-146, 2012.

[18] P. M. Villa, E. Kajantie, K. Räikkönen et al., "Aspirin in the prevention of pre-eclampsia in high-risk women: a randomised placebo-controlled PREDO Trial and a meta-analysis of randomised trials," British Journal of Obstetrics and Gynaecology, vol. 120, no. 1, pp. 64-74, 2013.

[19] A. Szarka, J. Rigó Jr., L. Lázár, G. Beko, and A. Molvarec, "Circulating cytokines, chemokines and adhesion molecules in normal pregnancy and preeclampsia determined by multiplex suspension array," BMC Immunology, vol. 11, article 59, 2010.

[20] J. C. P. Kingdom and S. Drewlo, "Is heparin a placental anticoagulant in high-risk pregnancies?" Blood, vol. 118, no. 18, pp. 4780-4788, 2011.

[21] W. Ramma and A. Ahmed, "Is inflammation the cause of preeclampsia?" Biochemical Society Transactions, vol. 39, no. 6, pp. 1619-1627, 2011.

[22] E. Groeneveld, M. J. Lambers, C. B. Lambalk et al., "Preconceptional low-dose aspirin for the prevention of hypertensive pregnancy complications and preterm delivery after IVF: a metaanalysis with individual patient data," Human Reproduction, vol. 28, no. 6, pp. 1480-1488, 2013.

[23] E. Schiff, E. Peleg, M. Goldenberg et al., "The use of aspirin to prevent pregnancy-induced hypertension and lower the ratio of thromboxane $\mathrm{A} 2$ to prostacyclin in relatively high risk pregnancies," New England Journal of Medicine, vol. 321, no. 6, pp. 351-356, 1989.

[24] A. Rahman and D. A. Isenberg, "Systemic lupus erythematosus," New England Journal of Medicine, vol. 358, no. 9, pp. 929-939, 2008.

[25] C. A. A. Silva, M. M. Leal, C. Leone et al., "Gonadal function in adolescents and young women with juvenile systemic lupus erythematosus," Lupus, vol. 11, no. 7, pp. 419-425, 2002.

[26] M. E. B. Clowse, M. Jamison, E. Myers, and A. H. James, "A national study of the complications of lupus in pregnancy," The American Journal of Obstetrics and Gynecology, vol. 199, no. 2, pp. 127.e1-127.e6, 2008.

[27] D. Erkan and L. Sammaritano, "New insights into pregnancyrelated complications in systemic lupus erythematosus," Current Rheumatology Reports, vol. 5, no. 5, pp. 357-363, 2003.

[28] K. Bramham, B. J. Hunt, S. Bewley et al., "Pregnancy outcomes in systemic lupus erythematosus with and without previous nephritis," Journal of Rheumatology, vol. 38, no. 9, pp. 1906-1913, 2011.

[29] E. F. Chakravarty, I. Colón, E. S. Langen et al., "Factors that predict prematurity and preeclampsia in pregnancies that are complicated by systemic lupus erythematosus," The American Journal of Obstetrics and Gynecology, vol. 192, no. 6, pp. 18971904, 2005

[30] M. J. Santos, D. Carmona-Fernandes, H. Canhão, J. Canas da Silva, J. E. Fonseca, and V. Gil, "Early vascular alterations in SLE and RA patients-a step towards understanding the associated cardiovascular risk," PLoS ONE, vol. 7, no. 9, Article ID e44668, 2012.

[31] S. Haque, M. Y. Alexander, and I. N. Bruce, "Endothelial progenitor cells: a new player in lupus?" Arthritis Research and Therapy, vol. 14, no. 1, article 203, 2012. 
[32] B. Santner-Nanan, M. John Peek, R. Khanam et al., "Systemic increase in the ratio between Foxp3+ and IL-17-producing CD4+ T cells in healthy pregnancy but not in preeclampsia," Journal of Immunology, vol. 183, no. 11, pp. 7023-7030, 2009.

[33] A. Becker-Merok, G. Ø. Eilertsen, and J. C. Nossent, "Levels of transforming growth factor- $\beta$ are low in systemic lupus erythematosus patients with active disease," Journal of Rheumatology, vol. 37, no. 10, pp. 2039-2045, 2010.

[34] C. K. Wong, L. C. W. Lit, L. S. Tam, E. K. M. Li, P. T. Y. Wong, and C. W. K. Lam, "Hyperproduction of IL-23 and IL17 in patients with systemic lupus erythematosus: implications for Th17-mediated inflammation in auto-immunity," Clinical Immunology, vol. 127, no. 3, pp. 385-393, 2008.

[35] A. Alunno, E. Bartoloni, O. Bistoni et al., "Balance between regulatory $\mathrm{T}$ and Th17 cells in systemic lupus erythematosus: the old and the new," Clinical and Developmental Immunology, vol. 2012, Article ID 823085, 5 pages, 2012.

[36] C. Tower, S. Mathen, I. Crocker, and I. N. Bruce, "Regulatory $\mathrm{T}$ cells in systemic lupus erythematosus and pregnancy," The American Journal of Reproductive Immunology, vol. 69, no. 6, pp. 588-595, 2013.

[37] K. Duckitt and D. Harrington, "Risk factors for pre-eclampsia at antenatal booking: systematic review of controlled studies," British Medical Journal, vol. 330, no. 7491, pp. 565-567, 2005.

[38] V. M. Abrahams, "Mechanisms of antiphospholipid antibodyassociated pregnancy complications," Thrombosis Research, vol. 124, no. 5, pp. 521-525, 2009.

[39] Q. Chen, F. Guo, S. Hensby-Bennett, P. Stone, and L. Chamley, "Antiphospholipid antibodies prolong the activation of endothelial cells induced by necrotic trophoblastic debris: implications for the pathogenesis of preeclampsia," Placenta, vol. 33, no. 10, pp. 810-815, 2012.

[40] J. A. Gómez-Puerta, R. Cervera, G. Espinosa et al., "Catastrophic antiphospholipid syndrome during pregnancy and puerperium: maternal and fetal characteristics of 15 cases," Annals of the Rheumatic Diseases, vol. 66, no. 6, pp. 740-746, 2007.

[41] Committee on Practice Bulletins-Obstetrics, American College of Obstetricians and Gynecologists, "Practice Bulletin No. 132: antiphospholipid syndrome," Obstetrics and Gynecology, vol. 120, no. 6, pp. 1514-1521, 2012.

[42] S. Wijetilleka, T. Scoble, and M. Khamashta, "Novel insights into pathogenesis, diagnosis and treatment of antiphospholipid syndrome," Current Opinion in Rheumatology, vol. 24, no. 5, pp. 473-481, 2012.

[43] M. Yurdakok, "Fetal and neonatal effects of anticoagulants used in pregnancy: a review," The Turkish Journal of Pediatrics, vol. 54, no. 3, pp. 207-215, 2012.

[44] B. Nørgård, E. Puhó, A. E. Czeizel, M. V. Skriver, and H. T. Sørensen, "Aspirin use during early pregnancy and the risk of congenital abnormalities: a population-based case-control study," The American Journal of Obstetrics and Gynecology, vol. 192, no. 3, pp. 922-923, 2005.

[45] T. G. Di Sessa, M. L. Moretti, A. Khoury, D. A. Pulliam, K. L. Arheart, and B. M. Sibai, "Cardiac function in fetuses and newborns exposed to low-dose aspirin during pregnancy," The American Journal of Obstetrics and Gynecology, vol. 171, no. 4, pp. 892-900, 1994. 


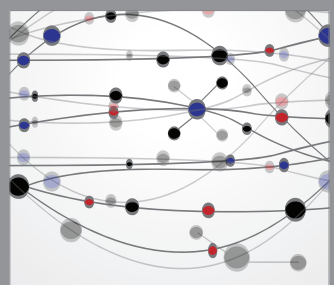

The Scientific World Journal
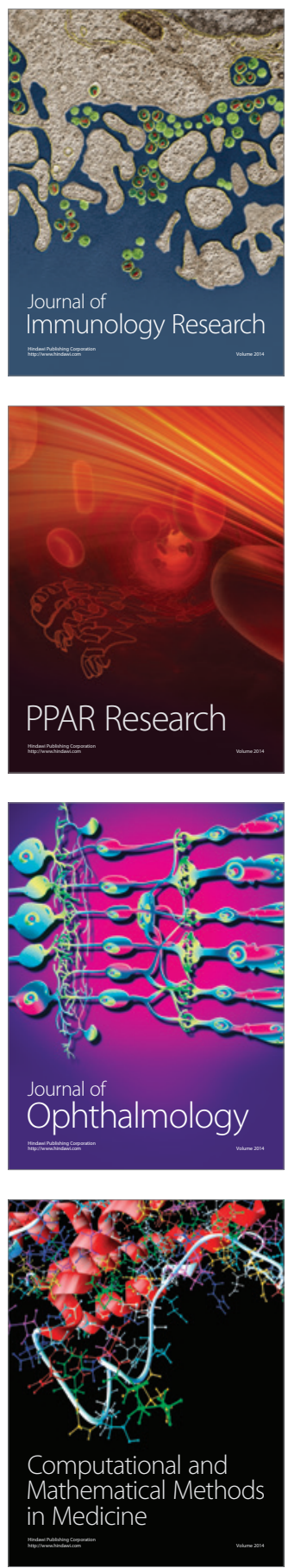

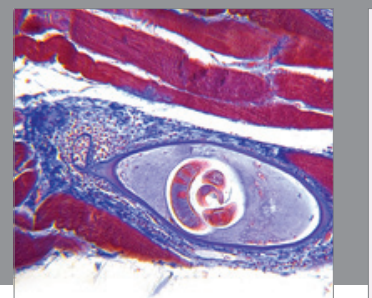

Gastroenterology

Research and Practice
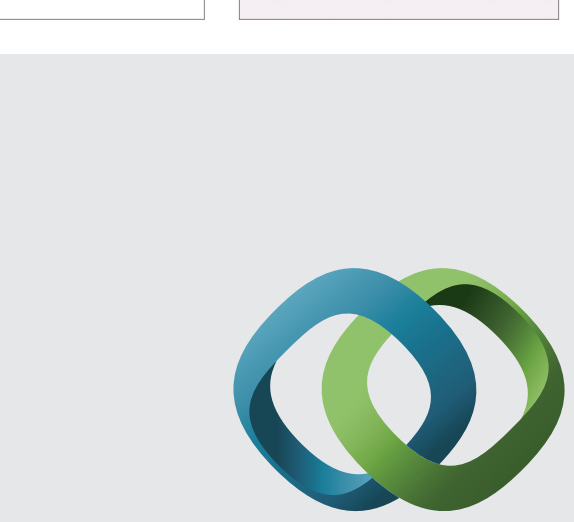

\section{Hindawi}

Submit your manuscripts at

http://www.hindawi.com
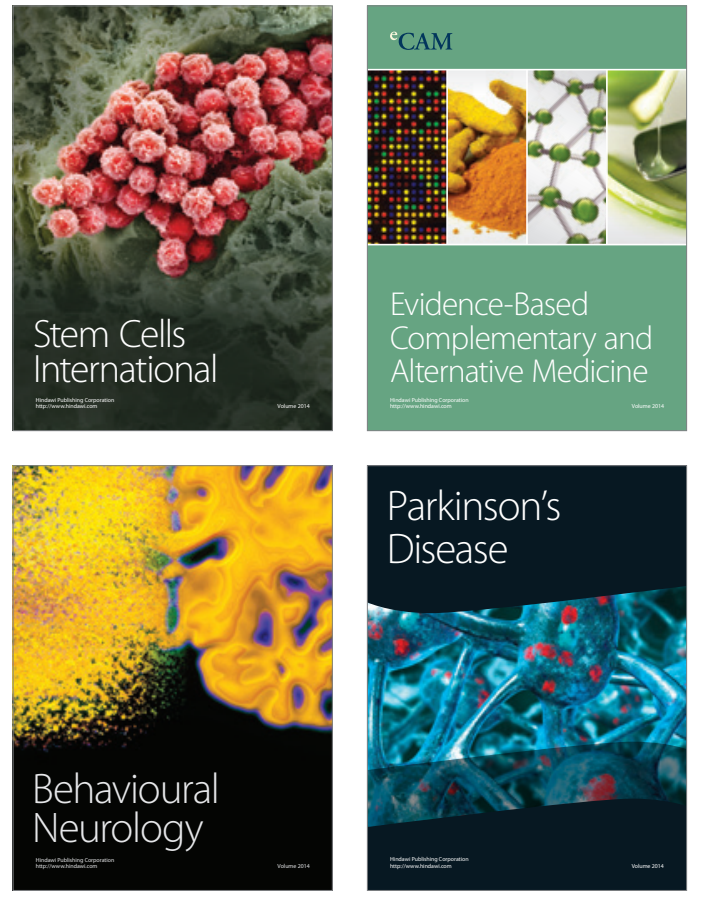
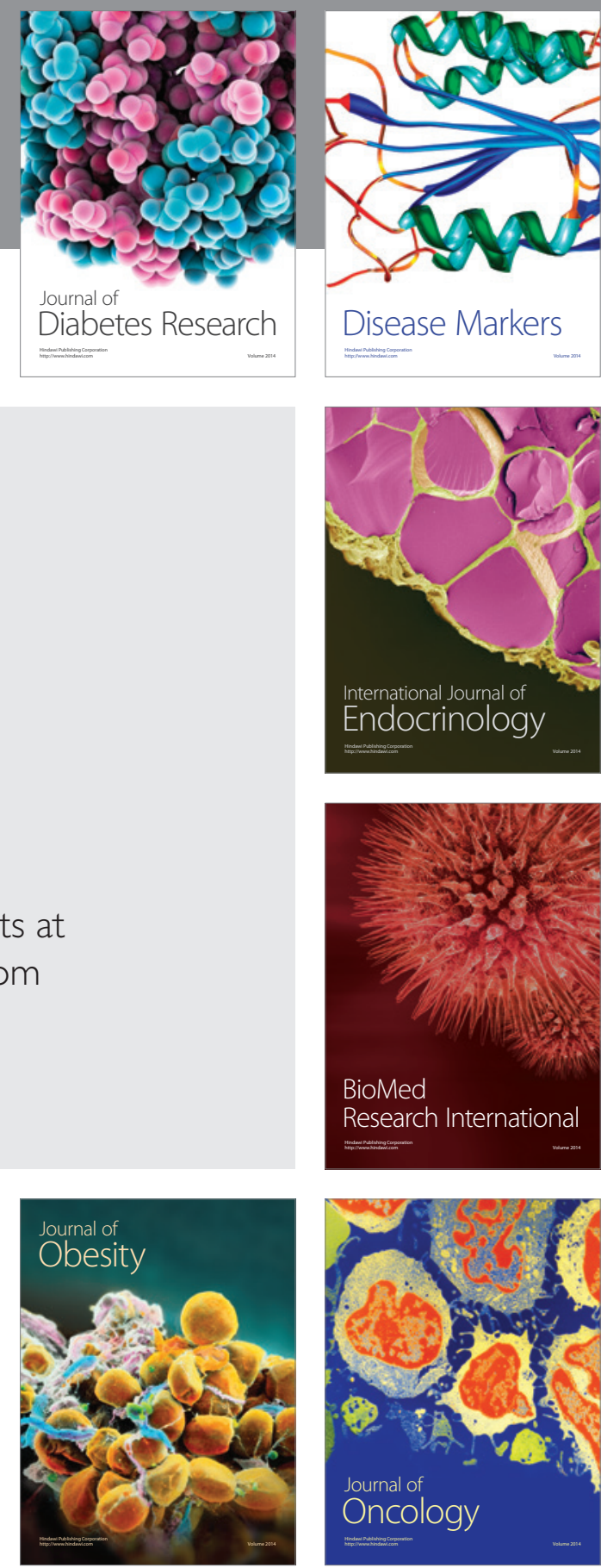

Disease Markers
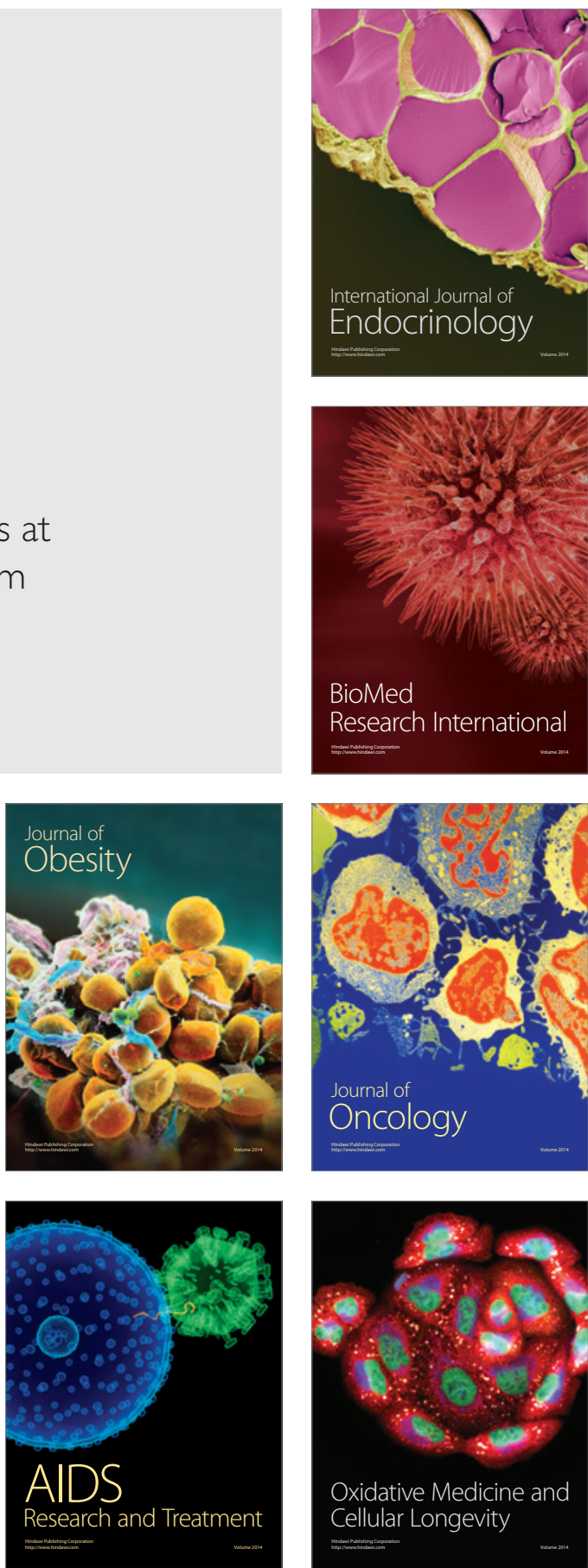